OPEN ACCESS

Edited by:

Salvatore Salomone,

University of Catania, Italy

Reviewed by:

Miguel A. Morales,

University of Notre Dame,

United States

Ana Gonçalves Domingos,

New University of Lisbon, Portugal

*Correspondence:

Kailash C. Pandey

kailash.pandey.nireh@gov.in

Specialty section: This article was submitted to Experimental Pharmacology and Drug Discovery,

a section of the journal

Frontiers in Pharmacology

Received: 06 March 2019

Accepted: 18 June 2019

Published: 18 July 2019

Citation:

Vardana, Dixit R, Tiwari $R$,

Katyal A and Pandey KC (2019)

Metacaspases: Potential Drug Target Against Protozoan Parasites.

Front. Pharmacol. 10:790. doi: 10.3389/fphar.2019.00790

\section{Metacaspases: Potential Drug Target Against Protozoan Parasites}

\author{
Vandana ${ }^{1,2}$, Rajnikant Dixit ${ }^{1}$, Rajnarayan Tiwari ${ }^{3}$, Anju Katyal ${ }^{2}$ and Kailash C. Pandey ${ }^{1,3 *}$ \\ ${ }^{1}$ Host-Parasite Interaction Biology Group, ICMR-National Institute of Malaria Research, New Delhi, India, ${ }^{2}$ Dr Ambedkar \\ Center for Biomedical Research, Delhi University, New Delhi, India, ${ }^{3}$ Department of Biochemistry, ICMR-National Institute for \\ Research in Environmental Health, Bhopal, India
}

Among the numerous strategies/targets for controlling infectious diseases, parasitesderived proteases receive prime attention due to their essential contribution to parasite growth and development. Parasites produce a broad array of proteases, which are required for parasite entry/invasion, modification/degradation of host proteins for their nourishment, and activation of inflammation that ensures their survival to maintain infection. Presently, extensive research is focused on unique proteases termed as "metacaspases" (MCAs) in relation to their versatile functions in plants and nonmetazoans. Such unique MCAs proteases could be considered as a potential drug target against parasites due to their absence in the human host. MCAs are cysteine proteases, having Cys-His catalytic dyad present in fungi, protozoa, and plants. Studies so far indicated that MCAs are broadly associated with apoptosis-like cell death, growth, and stress regulation in different protozoa. The present review comprises the important research outcomes from our group and published literature, showing the variable properties and function of MCAs for therapeutic purpose against infectious diseases.

Keywords: malaria, metacaspases, druggable target, proteases, plasmodium

\section{INTRODUCTION}

Studies over the past 15 years demonstrated that the proteases are not only essential for the maintenance of normal physiology but also play important roles in the regulation of cellular homeostasis and metabolism. Several proteases are considered as a potential drug target against the majority of infectious diseases. For an instance, antiretroviral drugs that inhibit viral integrase or GP41 (transmembrane protein) have been clinically approved to treat HIV infection (Arhel and Kirchhoff, 2010). Additionally, a broad network of protein-protein interactions was elucidated for treating various types of cancer (Kanhaiya et al., 2017) and other diseases like osteoporosis (Wnt proteins) (Rochefort, 2014). Similarly, several malaria parasite proteases have been largely targeted for their role in pathogenesis. Recently, plasmepsins (PMs), particularly PM-V, IX and X of Plasmodium falciparum, receive prime attention for exploring their potential as drug targets (Russo et al., 2010; Nasamu et al., 2017). Further, unique proteases called "metacaspases" are also presently focused for their role in programmed cell death (PCD) of the protozoan (Uren et al., 2000). However, MCAs are structurally related to metazoan caspases, having Cys-His catalytic dyad but possess different substrate specificity (Uren et al., 2000; Tsiatsiani et al., 2011). Metacaspases have a highly acidic S1 pocket leading to arginine and lysine specificity at the P1 position, in lieu of aspartic acid specificity for caspases (Mottram et al., 2003; 


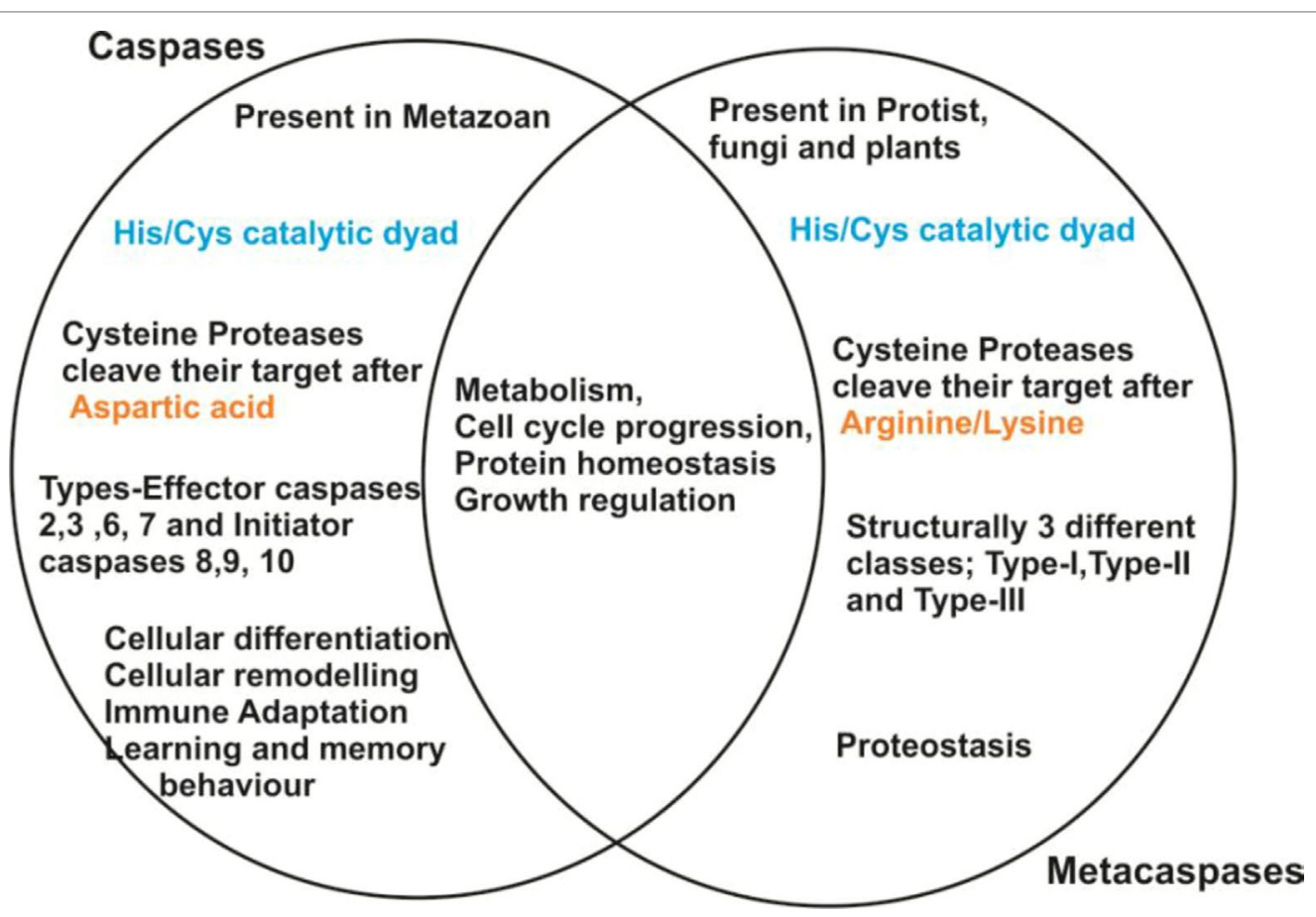

FIGURE 1 | Summarized pictorial representation showing the key properties of caspases and metacaspases.

Atkinson et al., 2009) (Figure 1). Further, paracaspases are also caspase-related proteins found in metazoans and Dictyostelium (Uren et al., 2000; Atkinson et al., 2009).

It has been proposed that metacaspases and paracaspases should be separated in a specific family of clan $\mathrm{CD}$. The basic topology of the caspases $(\mathrm{C} 14 \mathrm{~A})$ and paracaspases $(\mathrm{C} 14 \mathrm{~B})$ is found to be similar, whereas the topology of metacaspases (C14B) is quite different, suggesting that metacaspases were not similar with the caspases and paracaspases (Vercammen et al., 2004; Hachmann et al., 2012; Hulpiau et al., 2016). As per the present MEROPS classification, (Rawlings et al., 2017), all known cysteine proteases are categorized into 14 diverse clans according to their tertiary structure. Based on sequence similarity, proteases are clustered into families within each clan. Therefore, caspases, metacaspases, and paracaspases fit into the same clan of proteases (CD clan) and also to the same family (C14) based on the sequence similarity. The clan CD consists of cysteine-dependent proteases with a unique $\alpha / \beta$-fold called caspase-hemoglobinase (CHF) fold, which contains a large (p20) subunit having the catalytic histidine/cysteine dyad and a small (p10) subunit (Walker et al., 1994). There are three motifs in most of the conserved parts of the CHF fold: one at the $\mathrm{N}$-terminal $\beta$-strand, one before the catalytic histidine, and the other motif before the catalytic cysteine (Aravind and Koonin, 2002). The evolution of proteases from C14 family covered a long and complex series of speciation and duplication events that resulted in the significant variation in biochemical properties and hence a high degree of functional divergence. This notion was particularly true for metacaspases, which exhibited broader structural variation compared to caspases and paracaspases (Minina et al., 2017).

The comparative analysis of the different properties of caspases, metacaspases, and paracaspases reveals similarities and differences among them (Table 1).

\section{EVOLUTIONARY DIVERSITY OF METACASPASES-LIKE PROTEASES}

Evolutionary distribution of metacaspases among different phyla indicated that such proteases were evolved through endosymbiotic gene transfer (EGT) (Choi and Berges, 2013). Based on structural differences, metacaspases were subdivided into types I, II, and III. Type I metacaspases are defined by presence of an N-terminal extension and a zinc-finger motif (Vercammen et al., 2004), while type II category contains a linker domain, which separates the p20 and p10 domains. Moreover, type III metacaspases have unusual rearrangement of the two domains, with p10 domain located N-terminally to the catalytic p20 domain (Klemenčič and Funk, 2019). Although the present classification of metacaspases was based on the presence of structural features (e.g., an N-terminal domain for type I and a discrete linker region for type II metacaspases), various subtypes of metacaspases have arisen during evolution. For example, N-terminal domain of type I metacaspases is absent in prokaryotes and secondary endosymbionts, while the calcium-independent type II metacaspases comprise 
TABLE 1 | Comparative analysis of key properties of caspases, metacaspases, and paracaspases.

\begin{tabular}{|c|c|c|c|c|}
\hline Properties & Caspases & Metacaspases & Paracaspases & Reference \\
\hline Distribution & Metazoans & Protists, fungi, algae, and plants & Metazoans and Dictyostelium & Uren et al. (2000) \\
\hline Catalytic site & His-Cys catalytic dyad & $\begin{array}{l}\text { His-Cys catalytic dyad with few } \\
\text { exceptions such as TbMCA-1 } \\
\text { and TbMCA-4 that have Tyr and } \\
\text { Ser in place of His-Cys. }\end{array}$ & His-Cys catalytic dyad & $\begin{array}{l}\text { Uren et al. (2000); Hachmann et al. } \\
\text { (2012); McLuskey and Mottram } \\
\text { (2015) }\end{array}$ \\
\hline $\begin{array}{l}\text { Substrate } \\
\text { specificity }\end{array}$ & Aspartic acid specificity & $\begin{array}{l}\text { Arginine and lysine substrate } \\
\text { specificity }\end{array}$ & Arginine-specific protease & $\begin{array}{l}\text { Uren et al. (2000); Hachmann et al. } \\
\text { (2012) }\end{array}$ \\
\hline Types/forms & $\begin{array}{l}\text { Effectors caspases (caspase-2, } \\
-3,-6,-7) \text { and initiator caspases } \\
\text { (caspase-8, }-9,-10 \text { ) }\end{array}$ & $\begin{array}{l}\text { Type-I metacaspases have } \\
\text { N-terminal pro-domain with } \\
\text { proline-rich repeat motif and zinc } \\
\text { finger motif. } \\
\text { Type II metacaspases lack } \\
\text { pro-domain but possess a linker } \\
\text { region between the large (p20) } \\
\text { and small (p10) subunits. } \\
\text { Type III metacaspases found only } \\
\text { in algae that have undergone } \\
\text { secondary endosymbiosis }\end{array}$ & $\begin{array}{l}\text { Type-1 paracaspases constitute } \\
\text { MALT1-like domain having death } \\
\text { domain, immunoglobulin-like } \\
\text { domains and a caspase-like } \\
\text { domain. } \\
\text { Type-II paracaspases in metazoan } \\
\text { represent ancestral form, having } \\
\text { caspase-like-domain. }\end{array}$ & $\begin{array}{l}\text { Uren et al. (2000); Mottram } \\
\text { et al. (2003); Elmore (2007); } \\
\text { Hulpiau et al. (2016) }\end{array}$ \\
\hline $\begin{array}{l}\text { Biological } \\
\text { functions }\end{array}$ & $\begin{array}{l}\text { Key regulators of programmed } \\
\text { cell death, proliferation and } \\
\text { inflammation, playing essential } \\
\text { roles in the survival and death of } \\
\text { animal cells. }\end{array}$ & $\begin{array}{l}\text { Multifunctional proteases } \\
\text { essential for parasite physiology } \\
\text { but their detailed functions were } \\
\text { poorly characterized. }\end{array}$ & $\begin{array}{l}\text { Plays a major role in several pro- } \\
\text { inflammatory pathways in innate } \\
\text { and adaptive immunity. }\end{array}$ & $\begin{array}{l}\text { Nuñez et al. (1998) } \\
\text { Elmore (2007) }\end{array}$ \\
\hline $\begin{array}{l}\text { Enzymatic } \\
\text { functions }\end{array}$ & $\begin{array}{l}\text { Endo-proteases- hydrolyze } \\
\text { peptide bonds that depend on } \\
\text { catalytic cysteine residue in the } \\
\text { active site and occur after aspartic } \\
\text { acid residue in the substrates. } \\
\text { Caspase-mediated processing } \\
\text { results in substrate inactivation. } \\
\text { It may also generate active } \\
\text { signaling molecules that } \\
\text { participate in ordered processes } \\
\text { such as apoptosis and } \\
\text { inflammation. }\end{array}$ & $\begin{array}{l}\text { Cysteine proteases hydrolyze } \\
\text { peptide bonds after arginine/ } \\
\text { lysine residues in their substrates } \\
\text { Report on T. brucei MCA-4 } \\
\text { suggested that the phenotypes } \\
\text { induced by TbMCA- } 4 \text { expression } \\
\text { in yeast were completely lost } \\
\text { when the putative catalytic dyad } \\
\text { residues histidine }{ }_{164} \text { and cystein } \\
\text { were both independently mutated } \\
\text { to alanine. }\end{array}$ & $\begin{array}{l}\text { Cysteine proteases hydrolyze } \\
\text { peptide bonds after arginine } \\
\text { residues in their substrates }\end{array}$ & $\begin{array}{l}\text { 1. Uren et al. (2000); Szallies et al. } \\
\text { (2002); Mcllwain et al. (2013) }\end{array}$ \\
\hline
\end{tabular}

a shorter linker region between the p20 and p10 domains (Klemenčič and Funk, 2019). Type I and II metacaspases have been identified in plants based on their domain structures and similarities with metazoan "initiator" and "executioner" caspases. MCAs of type III were present only in algae that underwent secondary endosymbiotic processes; additional metacaspase-like proteases are reported in the Glaucophyta and Rhodophyta (Choi and Berges, 2013) and photosynthetic bacteria (Asplund-Samuelsson et al., 2012), indicating that they are the evolutionary ancestor of the advanced metacaspases. Further, metacaspase-like proteases in phytoplankton showed sequence homology with other metacaspases, but defies classification in conventional schemes; rather such metacaspase-like proteases exist in bacteria alongside a variant of type I metacaspases (Atkinson et al., 2009). Type II and III metacaspases were not detected in bacteria and they might be variants of bacterial type I metacaspases that evolved in plants and phytoplanktonic protists, respectively, during the establishment of plastids through the primary and secondary endosymbiotic events (Choi and Berges, 2013). From an evolutionary perspective, the existence of multiple metacaspases with structural and functional differences could be of great interest as it could be used to trace the origin of the multiple roles fulfilled by the closely related caspases in metazoans (Table 2 ).

\section{DISTRIBUTION AND FUNCTIONAL SPECIFICATION OF PARASITE METACASPASES}

The current ongoing research on metacaspases has brought information about their biological functions in protozoa and plants (Vercammen et al., 2004; Meslin et al., 2011; Rathore et al., 2015; Peña et al., 2017). Unequal distribution of metacaspases between different phyla is an important paradigm to describe their multi-functionality such as their role in cell death, stress regulation, growth and development of parasite, etc.

\section{Trypanosoma Metacaspases}

\section{Trypanosoma Brucei}

The genome of Trypanosoma brucei encodes five metacaspases: TbMCA1 to TbMCA5 were similar with type- metacaspases and did not exhibit any processing under normal growth condition of parasite (Kosec et al., 2006) (Figures 2-4). The three 
TABLE 2 | Structural-functional analyses of caspases, metacaspases, and paracaspases.

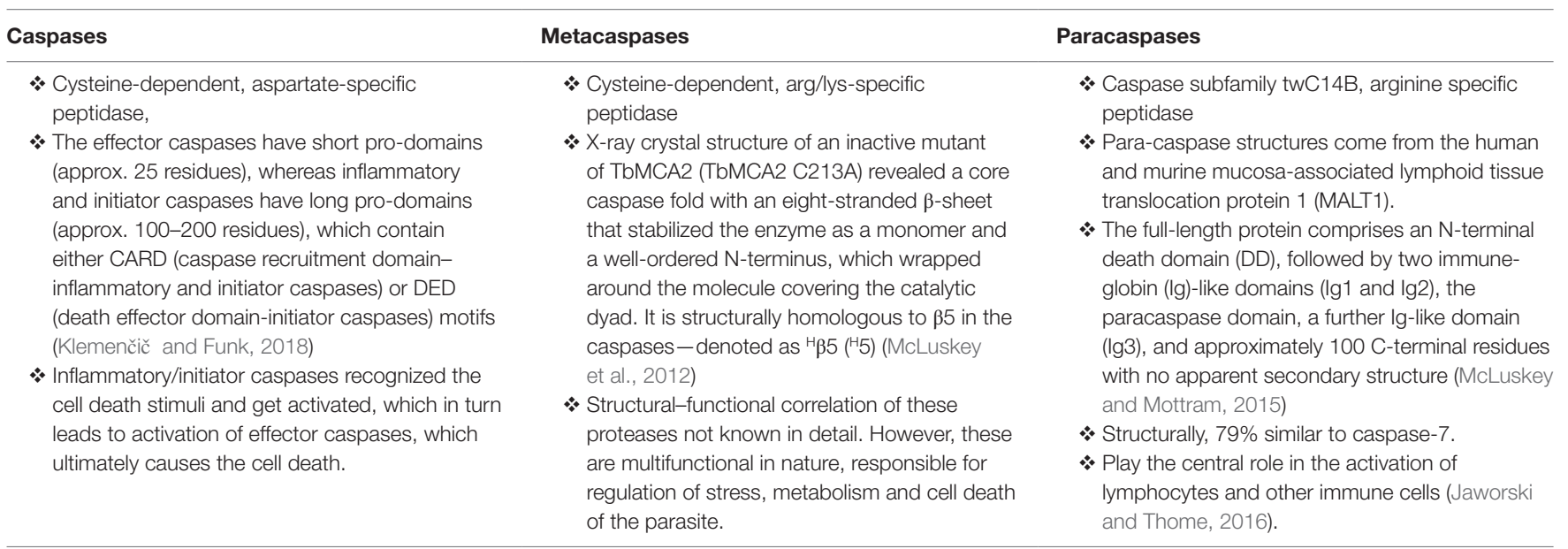

corresponding metacaspases genes (TbMCA-1, TbMCA-2, and TbMCA-4) of T. brucei seem to be absent in Trypanosoma cruzi. Studies suggested that TbMCA-2, TbMCA-3, and TbMCA-4 were maximally expressed in the bloodstream form of the parasite than in the procyclic form except TbMCA-5, which is expressed uniformly in both the forms of parasite life cycle (Meslin et al., 2011; Proto et al., 2011). A significant proportion of TbMCA-2, TbMCA-3, and TbMCA-5 were located predominantly in endosomes associated with RAB11 (Ras-like GTPase responsible for intracellular transport and cytokinesis) (Engstler et al., 2004; Helms et al., 2006; McLuskey and Mottram, 2015). There are three T. brucei metacaspases, TbMCA-2, TbMCA-3, and TbMCA-5, which are known to be active cysteine peptidases containing canonical histidine/cysteine dyad (Kosec et al., 2006; Moss et al., 2007), whereas TbMCA-1 and TbMCA-4 contain amino acid substitutions in their catalytic residues (histidine to tyrosine and cysteine to serine, respectively). On the other hand, a similar substitution of amino acid in TbMCA-4 resulted in a catalytically inactive pseudopeptidase, but it was still functional as a membrane-linked virulence factor, which was processed by TbMCA-3 (Meslin et al., 2011; Proto et al., 2011). The remaining genes (TbMCA-1 and TbMCA-4) were found to encode inactive proteases because they were predicted to have serine and tyrosine residues in place of catalytic cysteine and histidine residues, respectively (Meslin et al., 2011; Proto et al., 2011). Moreover, TbMCA-5 has unusual proline, glutamine, and tyrosine-rich $\mathrm{C}$-terminal extension in addition to its catalytic domain (Proto et al., 2011). On the other hand, TbMCA-2 and TbMCA-3 share $89 \%$ sequence similarity, differing only at the $\mathrm{N}$-terminus region. Studies on substrate specificity showed that TbMCA-2 has arginine/lysine specificity at the P1 position and enzyme activity was strictly $\mathrm{Ca}^{2+}$-dependent, requiring $1 \mathrm{mM}$ $\mathrm{CaCl}_{2}$ for maximum activity (Moss et al., 2007; Machado et al., 2013). Recent literature regarding the functionality of T. brucei metacaspases suggested that these proteases are likely to be involved in the regulation of PCD. For instance, Szallies et al. (2002) reported that the overexpression of TbMCA-4 in yeast cells causes phenotypic changes in terms of growth retardation, loss of clonogenicity followed by loss of respiration competence in the yeast cells in vitro (Szallies et al., 2002). However, the phenotypes induced by TbMCA-4 expression in the yeast cells were completely lost when the putative catalytic dyad residues histidine $_{164}$ and cystein ${ }_{218}$ were both independently mutated to alanine. Yeast cells expressing the respective alleles of TbMCA-4 were able to grow normally as the control (Szallies et al., 2002; Laverrière et al., 2012; McLuskey et al., 2014). This result clearly demonstrated that the effect of TbMCA- 4 depends on the putative catalytic dyad residues (Szallies et al., 2002; McLuskey et al., 2014). Further, studies speculated that TbMCA-2, TbMCA-3, and TbMCA-5 were involved in the regulation of cleavage furrow formation during cytokinesis of blood stream form (BSF) (Helms et al., 2006). However, the process of cytokinesis in BSF was not well illustrated, but it seems to be different from mammalian cells (Szallies et al., 2002; Machado et al., 2013). In addition, RNAi down-regulation study demonstrated that TbMCA-4 was essential for cell proliferation of the parasite (Meslin et al., 2011) (Figure 5). Further, TbMCA-4 processing by TbMCA-3 established an important link between two MCAs and that could be an indication of the existence of MCAs cascade similar to the mammalian caspases cascade system (McLuskey et al., 2014). The structural studies of TbMCA-2 suggested that metacaspases and caspases might be evolved independently from an ancestral metacaspase-like peptidase, with each family of enzymes evolving distinct activation mechanisms to regulate cell death pathways.

Conclusively, studies on T. brucei metacaspases suggested that its genome encodes MCA1-5 of which MCA-1 and MCA- 4 lack the predicted active site cysteine and probably are not active cysteine peptidase. However, it cannot yet be ruled out that MCA-1 or 4 might compensate for the lack of MCA-2, -3 , and -5 and carried out their role in mutant parasites. Indeed, it will be interesting to find out whether MCA-1 or -4 are up-regulated in MCA-2, -3 , and -5 knockout lines. Moreover, it is tempting to hypothesize that MCA-2, -3 , and -5 are involved in regulation of cleavage furrow formation during BSF cytokinesis, but little is known about the process of cytokinesis in BSF trypanosomes. Additionally, how the Trypanosome MCAs are directed to the compartment in which they reside is unclear. As per the literature, MCA-3 and MCA-5 are predicted to have signal peptides, whereas MCA-2 does not. 


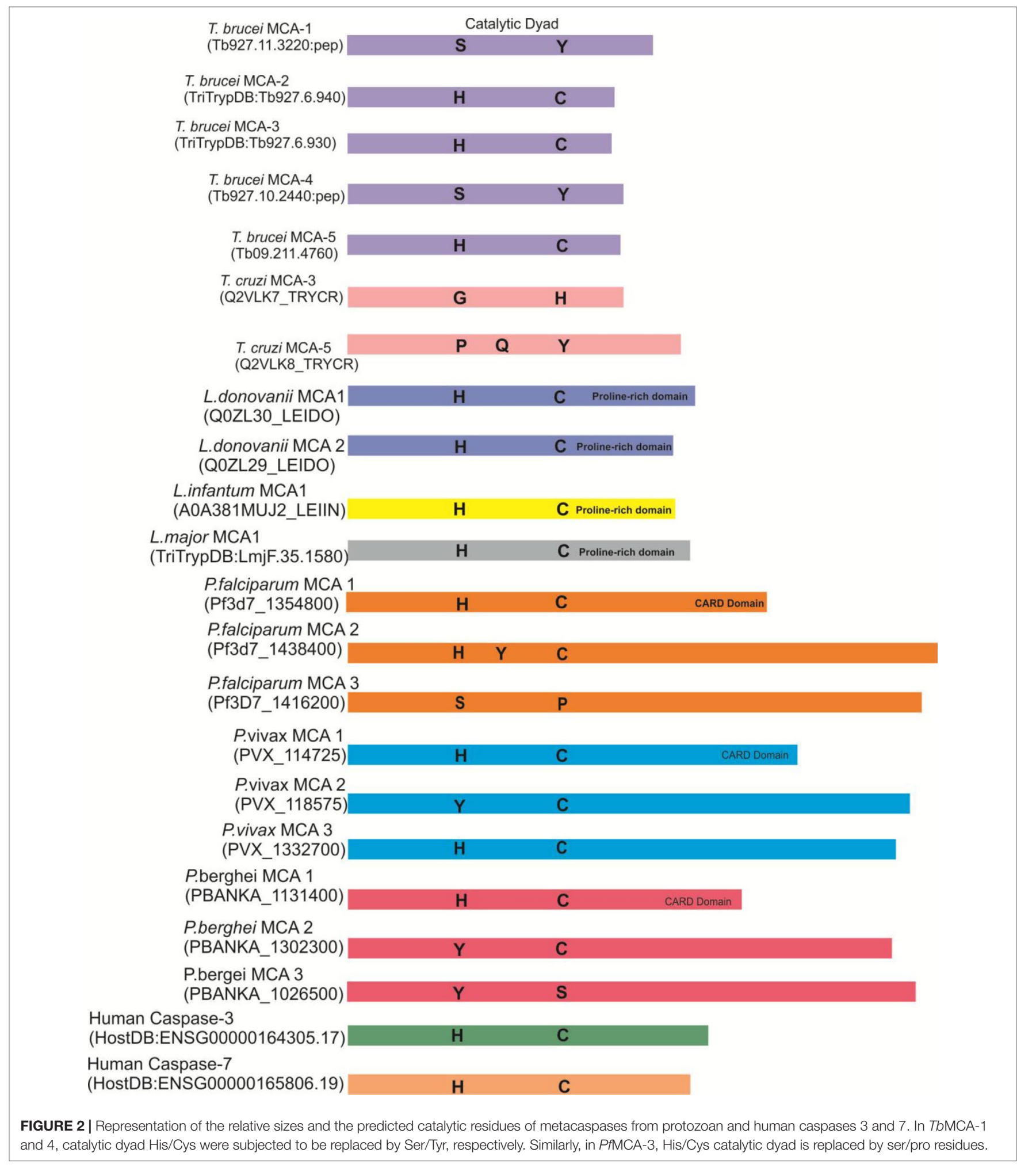

Therefore, MCA-3 and MCA-5 are likely to be sorted through the classic secretory pathway, while MCA-2 might either be trafficked in association with MCA-5, MCA-3, or any other ER directed protein, or through an alternative secretory pathway. Further, analysis of T. brucei MCAs is crucial to illuminate their function and to provide insights into the regulatory networks to which both active and inactive MCAs participate. Whether they might be responsible for PCD-like phenomena is an open question. Although it has been anticipated that metacaspases of yeast (González et al., 2007) and plants (Sundström et al., 2009) have caspase-like functions 


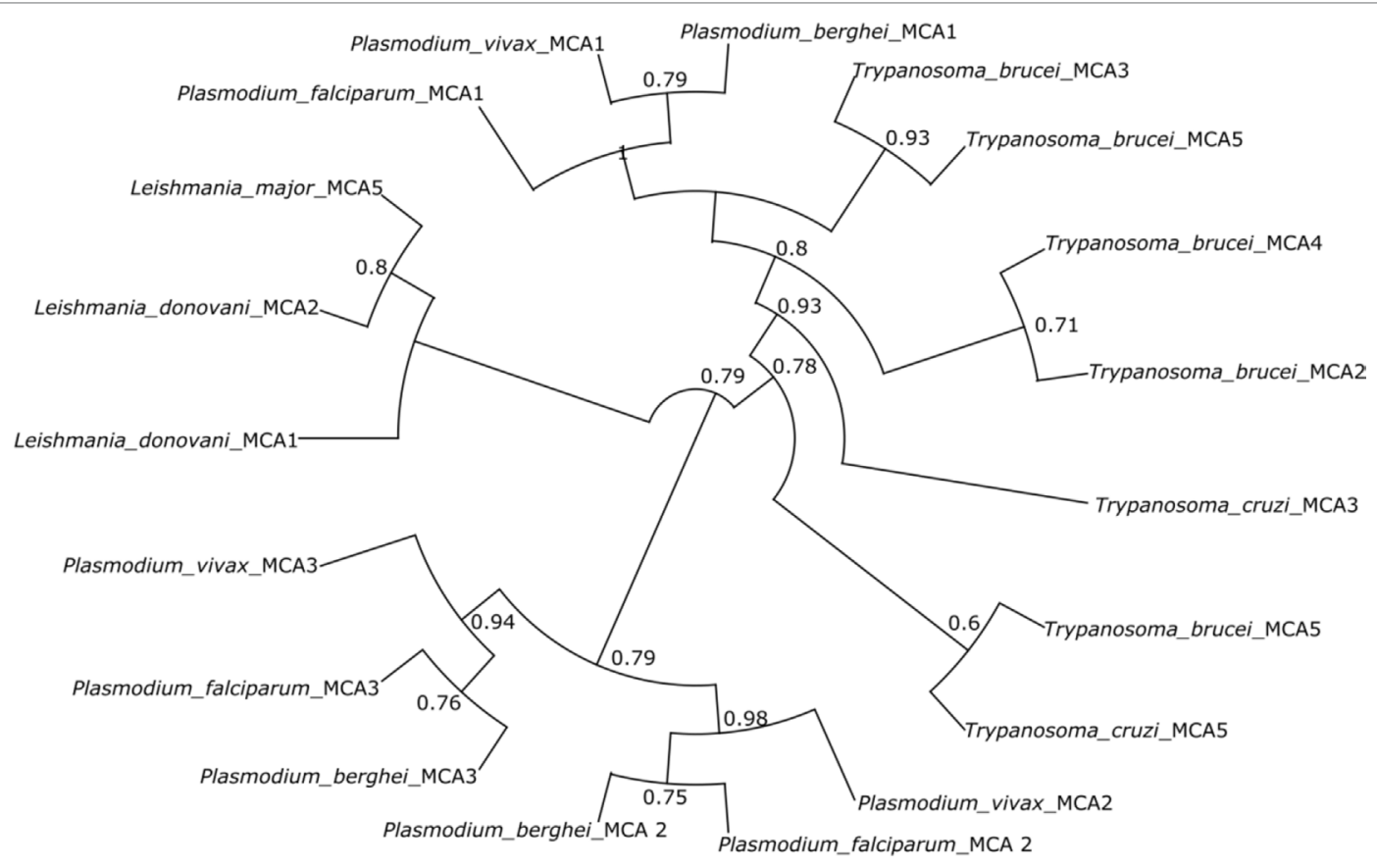

FIGURE 3 | Phylogenetic tree of metacaspases from protozoan parasites. The tree shows the distinct relationship between different metacaspases of the different parasites. The tree was constructed using the maximum parsimony method. Plasmodium metacaspases PfMCA-2, 3, PbMCA-2, 3, and PvMCA-2, 3 are phylogenetically distinct from metacaspases-1 of Plasmodium and other parasite metacaspases.

linked with PCD, there have not been any reported evidence for similar functions in BSF T. brucei. It cannot yet be ruled out that metacaspases are involved in apoptotic-like cell death of the $T$. brucei BSF; however, the data available suggest that metacaspases have PCD-independent functions that might be associated with RAB11-positive endosomes. However, metacaspases also occur in vesicles lacking RAB11; the significance and more research on this await elucidation.

\section{Trypanosoma cruzi}

In Trypanosoma cruzi, two metacaspase genes were reported, namely, TcMCA-3 and TcMCA-5. The metacaspase genes of T. cruzi were found to be homologous with TbMCA-3 and TbMCA-5 of T. brucei (Proto et al., 2011). Moreover, approximately 16 copies of TcMCA-3 and a single copy of TcMCA-5 per haploid genome have been reported. Further, the His/Cys catalytic dyad was present in both $T$. cruzi metacaspases, but TcMCA-3 showed a substitution of glycine $\rightarrow$ cysteine at the position next to the catalytic histidine residue. This glycine residue was broadly conserved among peptidases belonging to the clan CD; therefore, TcMCA-3 mutants with glycine instead of cysteine might be an inactive peptidase, and predicted to have an only regulatory function (Hulpiau et al., 2016). The association of TcMCAs with the RAB11 associated endosomes was not yet reported. Extending the role of T. cruzi metacaspases, there was indirect evidence suggesting that the metacaspases might be involved in PCD of the parasite (Helms et al., 2006). For an instant, epimastigotes over-expressing TcMCA-5 were more sensitive to fresh human serum (FHS)-induced PCD than the controls (Proto et al., 2011). In addition, TcMCAs also re-localized from the cytoplasm to the nucleus during apoptosis induced by FHS. Moreover, PCD was paralleled by an increase in peptidase activity against Z-YVAD-AFC (typical caspase substrate). The overexpression of TcMCA-3 in human cell line did not induce death or morphological changes typical of apoptosis (Alvarez et al., 2012). On the other hand, overexpression of TcMCA-5 in epimastigotes rendered them more susceptible to PCD, whereas TcMCA-3 overexpression is found to be lethal to parasite (Hulpiau et al., 2016). Studies reveal that the antagonistic activities of T. cruzi metacaspases affect the balance between cell proliferation, death, and differentiation of the parasite (Laverrière et al., 2012). Recently, de Castro et al. (2017) demonstrated that the procaspase-activating compound 1 (PAC-1) induces apoptosis in T. cruzi by interaction with TcMCA-3. The authors also revealed that the PAC-1 induces loss of cell viability, loss of mitochondrial potential, and externalization of phosphatidylserine (de Castro et al., 2017) (Table 3 and Figure 5). Conclusively, the direct involvement of TcMCA-3 and -5 in cell death is an open debate as it was evident that PCD in response to exposure of FHS occurred in T. cruzi. This could be beneficial in many ways, for example, by preventing an early inflammatory response if epimastigote enters the human bloodstream or could be rendered host macrophages more susceptible to invasion. Nevertheless, whether cell death processes in Trypanosoma parasite are mediated by MCA proteases and similar biochemical mechanisms as in metazoan still remains an open subject. 

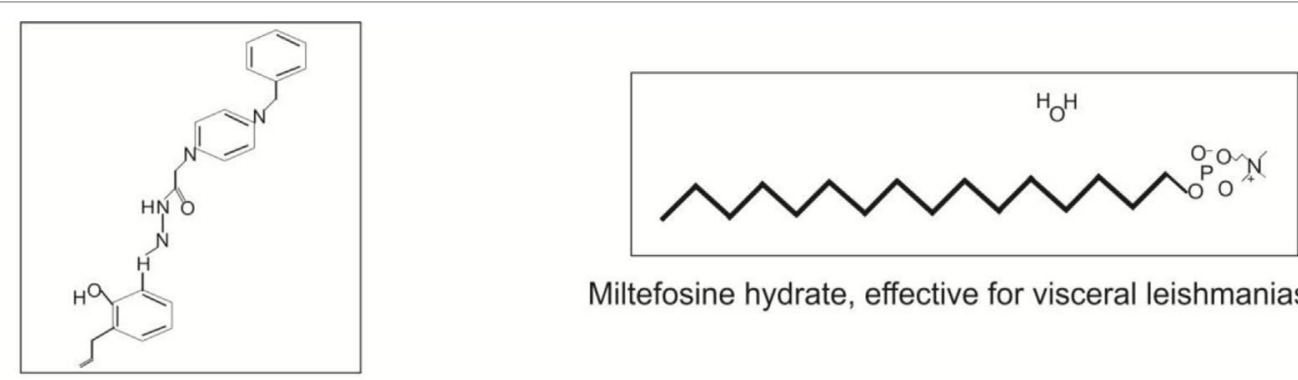

Miltefosine hydrate, effective for visceral leishmaniasis

Pro-caspase activating compound-1 (PAC-1) inducing

apoptosis in T.cruzi by interaction with TcMCA-3

(Emanuella de castro et al. 2017)

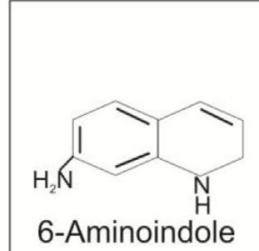

6-Aminoindole

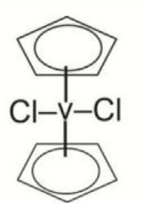

Bis(cyclopentadienyl) vanadium(IV)dichloride<smiles>O=C(O)c1cc(-c2ccc(C(=O)O)c(C(=O)O)c2)ccc1O</smiles>

Aurintricarboxylic acid

DiSB (Topoisomerase type I-B inhibitors) induced apoptosis primarly associated with up-regulation of metacaspses expression level in Leishmania (Chowdhary et al. 2011)<smiles>CC(C(=O)CF)N(C)C(=O)[C@H](Cc1ccccc1)NC(=O)OCc1ccccc1</smiles>

Z-FA-FMK analogues,inhibits PfMCA-2 activity \& expression and mediates parasite cell death via generation of oxidative stress.

(Vandana et al. 2018)

FIGURE 4 | Potential inhibitors/compounds used to target the different protozoan metacaspases, which could be further exploited as an effective drug target against infectious diseases.

\section{Leishmania Metacaspases}

All Leishmania species express one single metacaspase gene except L. infantum and L. donovani species where two metacaspases have been reported (Figures 2-4). Structurally, they possess conserved Cys-His catalytic dyad; they also share an N-terminal domain containing putative mitochondrial localization signal along with less conserved proline-rich C-terminal domain probably involved in protein-protein interactions (Lee et al., 2007; Choi and Berges, 2013). The presence of functional N-terminal mitochondrial localization signal in L. major indicated that MCAs might indirectly affect mitochondrion to trigger cell death (Zalila et al., 2011). In $L$. donovani, LdMCA-1 and LdMCA-2 were found to be expressed in both promastigote and axenic amastigote forms of the parasite, whereas MCA of L. mexicana was located in the mitochondria and associated with the mitotic spindle (Castanys-Muñoz et al., 2012). Enzymatically, LdMCAs displayed arginine/lysine substrate specificity without any auto-processing (Castanys-Muñoz et al., 2012). In contrast to LdMCAs, LmjMCA was activated by autoprocessing but has the same arginine/lysine substrate specificity (González et al., 2007). Importantly, in L. mexicana, deletion of MCA 
TABLE 3 | Comparative analyses of key features of protozoa metacaspases.

\begin{tabular}{|c|c|c|c|}
\hline Features & Leishmania metacaspases & Trypanosoma metacaspases & Plasmodium metacaspases \\
\hline Cys/His Catalytic dyad & All metacaspases contain His/Cys dyad. & $\begin{array}{l}\text { TbMCA2, TbMCA3, TbMCA5, TCMCA5 and } \\
\text { TcMCA3 possess His/Cys dyad } \\
\text { (McLuskey et al., 2014) }\end{array}$ & $\begin{array}{l}\text { MCA- } 1 \text { and }-2 \text { of } P \text {. vivax, } P \text {. falciparum and } \\
P . \text { berghi have His/Cys dyad. } \\
P V M C A-3 \text { has Cys/His catalytic dyad, but it } \\
\text { is absent in PfMCA-3 and PbMCA-3. }\end{array}$ \\
\hline $\begin{array}{l}\text { Localization/ } \\
\text { Expression }\end{array}$ & $\begin{array}{l}L d \mathrm{MC} 1 \text { and } L d \mathrm{MC} 2 \text { expressed in } \\
\text { promastigote and axenic amastigote forms. } \\
\text { LmjMCA located in the mitochondrion } \\
\text { and associated with the mitotic spindle } \\
\text { (Castanys-Muñoz et al., 2012). }\end{array}$ & $\begin{array}{l}\text { TbMCA-2, TbMCA-3, and TbMCA-4 } \\
\text { expressed in bloodstream stage only. } \\
\text { TbMCA-5 expressed in both life cycle } \\
\text { stages. } \\
\text { TbMCA-2, TbMCA-3, and TbMCA-5 were } \\
\text { located predominantly in endosomes } \\
\text { associated with RAB11. }\end{array}$ & $\begin{array}{l}\text { PfMCA-1 and PvMCA-1 gene expressed } \\
\text { during asexual stages (Meslin et al., } \\
\text { unpublished observations). } \\
\text { PbMCA-1 expression was detected in female } \\
\text { gametocytes, oocysts, and sporozoites. } \\
\text { PfMCA-2 was localized in schizonts and } \\
\text { gametocytes stages I-IV (Vandana et al., } \\
\text { 2018). }\end{array}$ \\
\hline $\begin{array}{l}\text { Enzymatic Activation/ } \\
\text { Processing }\end{array}$ & $\begin{array}{l}\text { LdMCAs has arginine/lysine specificity } \\
\text { without any proteolytic activation } \\
\text { LmjMCA activated by auto-processing and } \\
\text { shows arginine/lysine substrate specificity. }\end{array}$ & $\begin{array}{l}\text { Arg/Lys specificity and activity was strictly } \\
\mathrm{Ca}^{2+}-\text { dependent. }\end{array}$ & $\begin{array}{l}\text { Arg/Lys specificity and activity were } \mathrm{Ca}^{2+} \text { - } \\
\text { independent and no auto-processing } \\
\text { occurred in PfMCA-2 (Vandana et al., 2018). } \\
\text { PfMCA-1 shows auto processing leading } \\
\text { to pro-domain removal as typical of initiator } \\
\text { caspases. }\end{array}$ \\
\hline Forms/Types & $\begin{array}{l}\text { All Leishmania species expressed one } \\
\text { single metacaspase gene except } L \text {. } \\
\text { infantum and } L \text {. donovani, in which two } \\
\text { metacaspases were reported. }\end{array}$ & $\begin{array}{l}\text { Type-I metacaspases; T. brucei has } \\
\text { TbMCA1-TbMCA5 and T. cruzi has } \\
\text { TCMCA3 and TCMCA5. }\end{array}$ & $\begin{array}{l}\text { Type-I metacaspases; P. falciparum PfMCA1- } \\
3 \text {, P. vivax PVMCA1-3, and P. berghei } \\
\text { (PbMCA1-3) }\end{array}$ \\
\hline Predicted Functions & $\begin{array}{l}\text { Predicted to be involved in stress-induced } \\
\text { cell death regulation. } \\
\text { LmjMCAs involved in cell cycle progression. }\end{array}$ & $\begin{array}{l}\text { Regulation of cell death pathways; } \\
\text { cytokinesis of parasite. } \\
\text { Mechanistic role in PCD needs to be } \\
\text { explored deeply. }\end{array}$ & $\begin{array}{l}\text { PfMCA-2 is likely involved in the progression } \\
\text { of growth in vitro and indirectly responsible } \\
\text { for cell death in the parasite (Vandana et al., } \\
2018 \text { ). } \\
\text { Role of other MCAs of } P \text {. vivax and P. berghei } \\
\text { needs to be elucidated. }\end{array}$ \\
\hline
\end{tabular}

did not affect the cell growth and viability of procyclic promastigotes (González et al., 2007). Further, overexpression of MCAs in amastigotes resulted in lower replication rate, which suggested that MCAs act as an amastigotes-specific growth suppressor.

Reports about the involvement of MCAs in Leishmania cell death reveal that $L m j \mathrm{MCA}$ induces cell death in parasite, either by releasing its catalytic domain or by interaction of the C-terminal domain with partners involved in stress regulation or cell death in the presence of different apoptotic stimuli (miltefosine, curcumin, and $\mathrm{H}_{2} \mathrm{O}_{2}$ ) (Khademvatan et al., 2011; Casanova et al., 2015; Peña et al., 2017). Experimental evidence also suggested that the process of autophagy occurred in low nutrient concentration, which resulted in overexpression of LmjMCA (Khademvatan et al., 2011). Further, $L m j$ MCA overexpression was found to enhance L. major sensitivity to oxidative stress compared to wild-type parasites expressing the endogenous metacaspases at optimum levels (Dolai et al., 2011). Report by Chowdhury et al. (2014) further suggested that the DiSB (topoisomerase type I-B inhibitor) induced apoptosis appears to be primarily associated with up-regulation in the expression level of metacaspases and generation of oxidative stress in L. donovani (Chowdhury et al., 2014). Interestingly, Shadab et al. (2017) group recently reported that peptide ecotin-like ISP3 of $L$. major specifically binds to MCAs and interferes with its trypsin-like activity in presence of heat shock, thereby significantly reducing parasite cell death (Shadab et al., 2017).

The endoplasmic reticulum-induced stress leads to the occurrence of $\mathrm{Ca}^{+2}$-dependent apoptosis-like cell death. However, cell death in ER stress-induced Leishmania cells was mediated by the mitochondrial apoptotic pathway. This pathwar involved ROS production, cytosolic $\mathrm{Ca}^{+2}$ imbalances, mitochondrial depolarization, and ultimately the release of Endo $G$ from mitochondria to the nucleus via the cytoplasm (Dolai et al., 2011; Khademvatan et al., 2011). In addition, Khademvatan et al. (2011) report also suggested that the phenomenon of PARP cleavage (DNA repair enzyme that catalyzes the polyADP-ribosylation of various nuclear proteins), a prominent feature of metazoan apoptosis, also occurred in Leishmania (Khademvatan et al., 2011). Moreover, it was shown by a two-hybrid system that L. major mitogen-activated protein kinase MPK7 and calpain interact with the C-terminal domain of MCA, which probably causes induction of parasite death (Khademvatan et al., 2011; Shadab et al., 2017). Interestingly, miltefosine [hexadecylphosphocholine (HePc)] was found to be effective for visceral leishmaniasis and responsible for cleavage of PARP-like protein during apoptosis in Leishmania treated with $\mathrm{H}_{2} \mathrm{O}_{2}$. This process was blocked by caspase inhibitors (Khademvatan et al., 2011) (Table 3 and Figure 5).

Studies revealed that LmjMCA is involved in autophagy in relation with over-expression of the gene and interaction of LmjMCA, mainly owing to its C-terminal domain, with itself and other proteins. These findings open new perspectives on the function of the MCA. The identification of the enzymatic substrates of LmjMCA and cell death triggering stimuli would clarify the metabolic pathways involving LmjMCA mediated cell death and/or autophagy. An important aspect of investigating metacaspases-like proteases in Leishmania is the uncertainty 


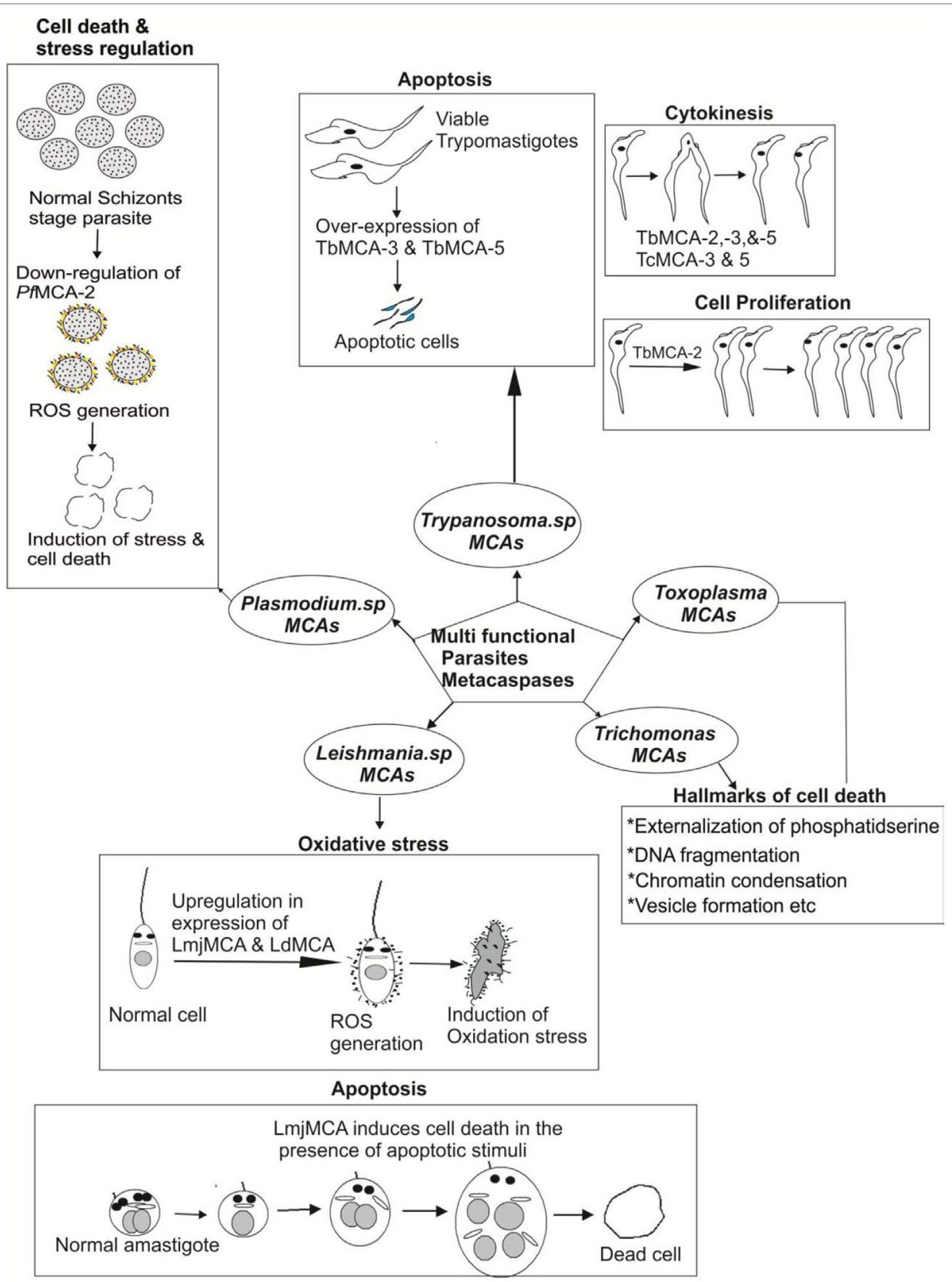

FIGURE 5 | Pictorial depiction for multi-functions of metacaspases in different parasites: Trypanosoma metacaspases are found to play versatile function such as apoptosis, cell proliferation, cytokinesis of the parasites (Meslin et al., 2011; Proto et al., 2011); Leishmania metacaspases are involved in stress regulation and autophagy of the parasite (Khademvatan et al., 2011; Casanova et al., 2015; Peña et al., 2017); Plasmodium metacaspases also play a role in apoptosis and stress regulation of the parasites (Vandana et al., 2018).

in autophagy/PCD relationships, which further leaves an open question about the mechanistic intersections between the two processes (Casanova et al., 2015; Chowdhury et al., 2014). Moreover, report by Castanys-Munoz et al. (2012) depicted that MCAs were not involved in Leishmania proliferation; rather, they act as amastigotes-specific growth suppressors, thereby regulating the parasite proliferation in the host. This self-regulation of growth is likely to be essential in maintenance of the infection thus greatly contributing to pathogenesis. Furthermore, stress-induced cell death was reported, which might be facilitated by the release of lysosomal enzymes after the disruption of organelle, and this could involve the cleavage of MCAs by the released cathepsin-like cysteine proteases. Conclusively, detailed studies on Leishmania metacaspases in context with the induction or inhibition route of apoptosis are an important subject to explore in depth. 


\section{Plasmodium Metacaspases}

Among the potential druggable targets against malaria, Plasmodium "metacaspases" are recently emerging as a potent candidate to explore. Till today, three MCAs were reported in the Plasmodium genomes: P. falciparum (PfMCA1-3) and P. vivax (PvMCA1-3) and in the murine parasite P. berghei (Atkinson et al., 2009) (PbMCA1-3) (Figures 2-4). Proteins having caspase-like activity were identified in the cytoplasm of the ookinete, and more than $50 \%$ of the mosquito midgut stages of the parasite die naturally by apoptosis before the gut invasion (Meslin et al., 2007). Study by Picot et al. (1997) suggested that the PfMCA-1 possesses the His-Cys catalytic dyad and upstream signaling pathways such as death domain or CARD, a module of 90-100 amino acids involved in apoptosis signaling pathways. The PfMCA1-CARD consists of 76 amino acid residues that contributed the highest sequence similarity with caspase-1 (20.4\%) of mice and humans (Meslin et al., 2007; Mutai and Waitumbi, 2010). Apoptosis-like DNA fragmentation/degradation in P. falciparum after chloroquine treatment in vitro was reported for the first time (Picot et al., 1997). Further, caspase-3 like subfamily member was associated with $P$. berghei ookinete apoptosis showing prominent features such as chromatin condensation, DNA fragmentation, and externalization of phosphatidylserine (Al-Olayan et al., 2002; Elmore, 2007). Moreover, in the presence of natural sunlight, there was an inhibition of parasite growth in vitro, which in turn leads to cell death in late trophozoites and schizonts (Engelbrecht and Coetzer, 2015). A study by Rathore et al. (2015) reported the occurrence of apoptosis-like cell death persuaded by cellular stress and organelle dysfunction, which was further attributed to the disruption of cellular homeostasis in P. falciparum. This report also demonstrated that the consistent stress on parasite led to the activation of Z-VAD-FMK-binding proteases and raised the cytosolic calcium levels along with the loss of mitochondrial membrane potential in parasites (Rathore et al., 2015). Activation of Z-VAD-FMK-binding proteases leads to degradation of the phylogenetically conserved protein, TSN (Tudor staphylococcal nuclease), a known target of metacaspases, along with the degradation of other components of the spliceosomal complex (Rathore et al., 2015). These findings are a clear indication for the existence of caspase-like proteases, which are known as "metacaspases" in Plasmodium, and they might have a role in PCD or in the regulation of growth and development of parasites (Ch'Ng et al., 2010; Rathore et al., 2015). However, the direct roles of these metacaspases are still not known in the malaria parasite (Shrestha and Megeney, 2012). Therefore, these proteases need to be explored in order to address their effective therapeutic potential against malaria.

In continuation of the above-mentioned reports on Plasmodium metacaspases, we find that $P$. falciparum MCA-2 (PfMCA-2) have Arg/Lys substrate specificity at $\mathrm{pH} 7.4$ (Vandana et al., 2018). A multiple sequence alignment of particular PfMCA-2 has conserved tyrosine residues near to its cysteine and histidine catalytic dyad, which might be involved in substrate recognition. Unlike common malarial cysteine proteases such as falcipains, PfMCA-2 cleaved neither hemoglobin nor BSA or casein-like macromolecular substrates. The effector caspases inhibitor Z-FAFMK remarkably inhibits the PfMCA-2 activity and the in vitro progression of $P$. falciparum (Vandana et al., 2018). Moreover, Z-FA-FMK also induces the oxidative stress by generating reactive oxygen species, which in turn is responsible for the occurrence of parasite cell death (Vandana et al., 2018) (Figures 5 and 6). This information could be important for further exploring the detailed function of metacaspases in Plasmodium (Table 3 and Figure 6).

In summary, the studies done so far on this subject revealed that Plasmodium metacaspases might play a role in regulation of stress-dependent PCD and growth of the parasite.

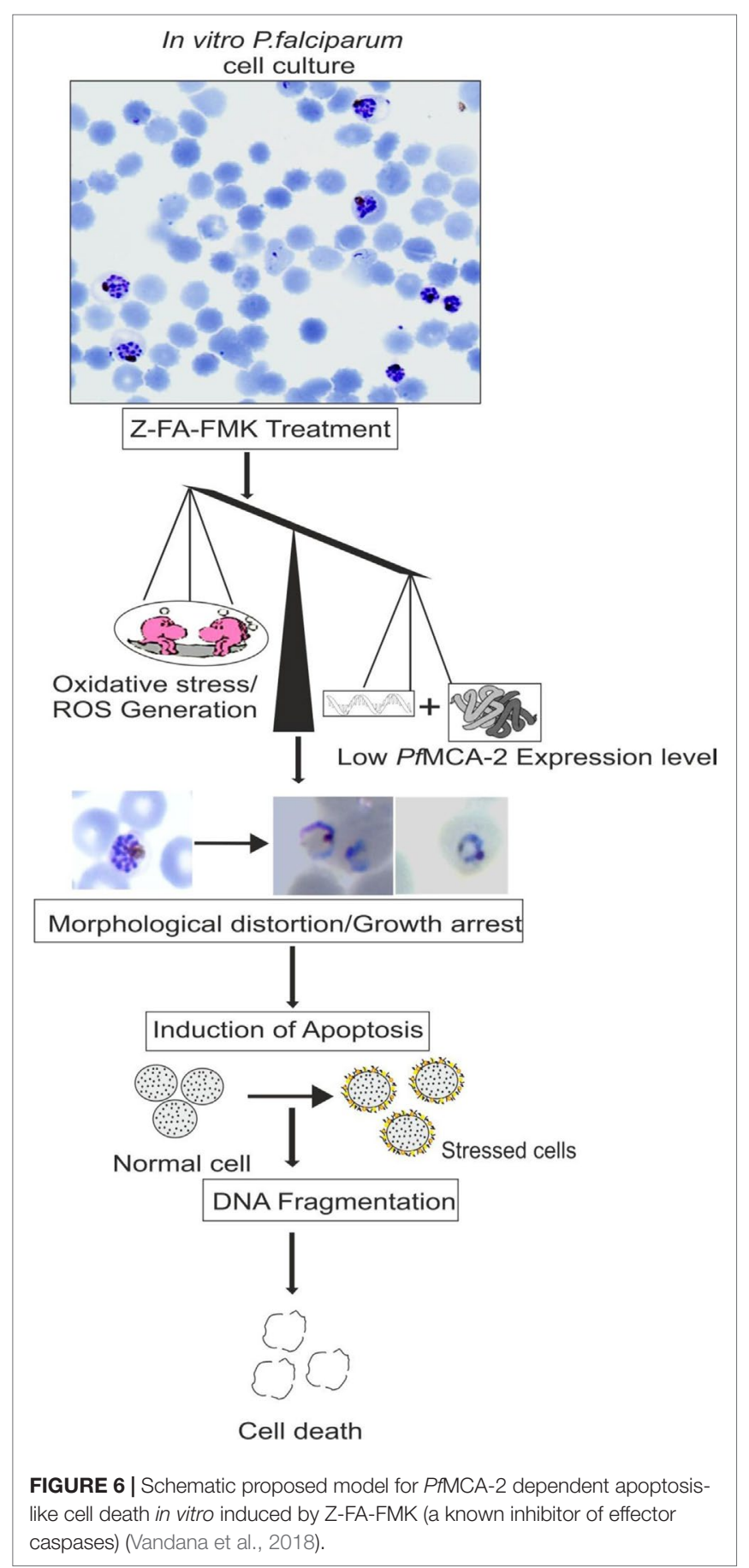


However, Plasmodium metacaspases are not well studied in terms of their structural-functional prospects; therefore, more extensive research is needed in order to target such proteases for antimalarial drug discovery.

\section{Other Protozoan Parasite and Diatom Metacaspases}

There are several studies that emphasize the occurrence of apoptosis-like cell death in other protozoan parasites. For instance, induction of apoptotic-like morphological changes in response to oxidative stress was observed in Giardia, Trichomonas vaginalis, Entamoeba histolytica, and Blastocystis. Bagchi et al. (2012) performed bioinformatics survey of Giardia genomes and reported key genes involved in autophagy (Bagchi et al., 2012). However, in the same study, the authors did not find any apoptotic gene that had significant similarity with identified apoptotic genes of other protozoa and eukaryotes except TOR and ATG8 (Bagchi et al., 2012). Moreover, the caspaselike activity in Giardia cell-free lysates was absent indicating the absence of caspase-like proteases in Giardia genome (Dubios et al., 2008; Bagchi et al., 2012). In Toxoplasma gondii, three metacaspases genes were annotated (TGGT1_206490, TGGT1_278975, and TGGT1_243298) and their respective proteins belong to ICE family (Li et al., 2016). Interestingly, only TGGT1_206490 carries histidine and cysteine catalytic dyad as reported for clan CD. However, such catalytic dyad was absent in the other two metacaspases. Study by Li et al. (2016) revealed that the in vitro growth and in vivo virulence of T. gondii were not affected in TgMCA knockout parasite. However, there was a significant decline in apoptotic cell death in TgMCA knockout parasites indicating TgMCA plays an important role in T. gondii cell death (Li et al., 2016). Further, Trichomonas vaginalis also encodes metacaspases gene (TVAG_344040), but there are limited studies focusing its role in the cell death of the parasite (Chose et al., 2002). However, characteristic features of cell death including DNA fragmentation, chromatin condensation, nuclear fragmentation, vesicle formation, externalization of phosphatidylserine, vacuolization, etc. were reported in both T. vaginalis and $T$. foetus along with the typical caspase-like activity in T. foetus (Chose et al., 2002; Arroyo et al., 2015). Additionally, in Blastocystis hominis, caspase-3 like protease influenced the cell death, but it was not essential for the occurrence of DNA fragmentation/apoptosis (Nasirudeen and Tan, 2004). Moreover, the process of apoptosis in this unicellular parasite was associated with mitochondrial dysregulation. Similarly, the calpain-like protease was found to be responsible for inducing cell death in Entamoeba histolytica (DomínguezFernández et al., 2018). Further, metacaspases-5 from the model diatom Phaeodactylum tricornutum (PtMC5) possess calciumdependent protease activity. This activity included autoprocessing and cleavage after arginine residue (Van Creveld et al., 2018). Such type III metacaspase also controls cell death in a marine diatom. A study by Bidle and Bender (2008) further suggested that iron starvation leads to activation of metacaspases and PCD in the marine diatom Thalassiosira pseudonana (Bidle and Bender, 2008).
The main aim of focusing PCD associated proteins in protists is to target them for the development of a potential drug against the parasite-based infectious diseases. Therefore, at present, it is very important to explore the functional properties of such proteases for the elucidation of their functional relationship with metazoan caspases.

\section{TUDOR STAPHYLOCOCCAL NUCLEASE AS A NATURAL SUBSTRATE OF METACASPASES}

Unraveling the identity of natural substrates of metacaspases is important for revealing the molecular mechanisms of metacaspasedependent processes. Presently, TSN is the only protein found to be cleaved by metacaspases in vivo (Gutierrez-Beltran et al., 2016). TSN is an evolutionarily and structurally conserved protein found in all eukaryotes (except for budding yeast). Functionally, TSN is involved in numerous fundamental mechanisms of gene regulation in animal cells, including transcription, mRNA splicing, and RNA silencing (Carmona-Gutierrez et al., 2010; Gutierrez-Beltran et al., 2010; Tsiatsiani et al., 2011). A report by Sundström et al. (2009) suggested that the reduced expression level of TSN causes cell death (Tsiatsiani et al., 2011; Bagchi et al., 2012) and impairs plant viability and stress tolerance (Carmona-Gutierrez et al., 2010). Moreover, active type II metacaspase from Norway spruce (mcII- $\mathrm{Pa}$ ) causes fragmentation of endogenous TSN during developmental and oxidative stress-induced cell death. Further, caspase-3-mediated cleavage of human TSN at the cleavage site DAVD/S (Pop and Salvesen, 2009; Carmona-Gutierrez et al., 2010) has been revealed in cells undergoing apoptotic-like cell death. Further, Sundström et al. (2009) have demonstrated that TSN imparts stress tolerance in Arabidopsis through selective stabilization of mRNAs-encoding secreted proteins (Sundström et al., 2009). Notably, a significant proportion of these proteins are cysteine and serine protease inhibitors, which are known to suppress cell death in plants (Sundström et al., 2009; Dit Frey et al., 2010). But the mechanism of TSN-dependent stabilization of specific mRNAs remains unknown. Studies on the fragmentation of TSN by effector caspases and type-II metacaspase suggested that the animals and plants may have some common key proteolytic pathways responsible for the normal cell viability and physiology. A study by Rathore et al. (2015) suggested that PfTSN harbors the DFVD motif near its C-terminus, which is a probable cleavage site for caspase-like enzymes (Figure 7). In the same study, authors have suggested that activation of caspaselike proteases leads to the reduction of the PfTSN level in parasites along with other associated nuclear proteins like PfSmD1 and PfSmD3, which are part of nuclear splicing machinery (Rathore et al., 2015). We also found that PfMCA-2 interacts specifically with the Tudor domain of the PfTSN (Vandana et al., 2018). Further, the molecular mechanism of TSN degradation by Plasmodium MCA-2 and its consequence to the apoptosis pathway will be an interesting area to address in the near future. The existence of TSN and MCA interaction was not reported yet in Leishmania and Trypanosoma. Moreover, the correlation between metacaspasemediated fragmentation of endogenous TSN and abolishment of its fragmentation by MCAs in the presence of inhibitor is an important 

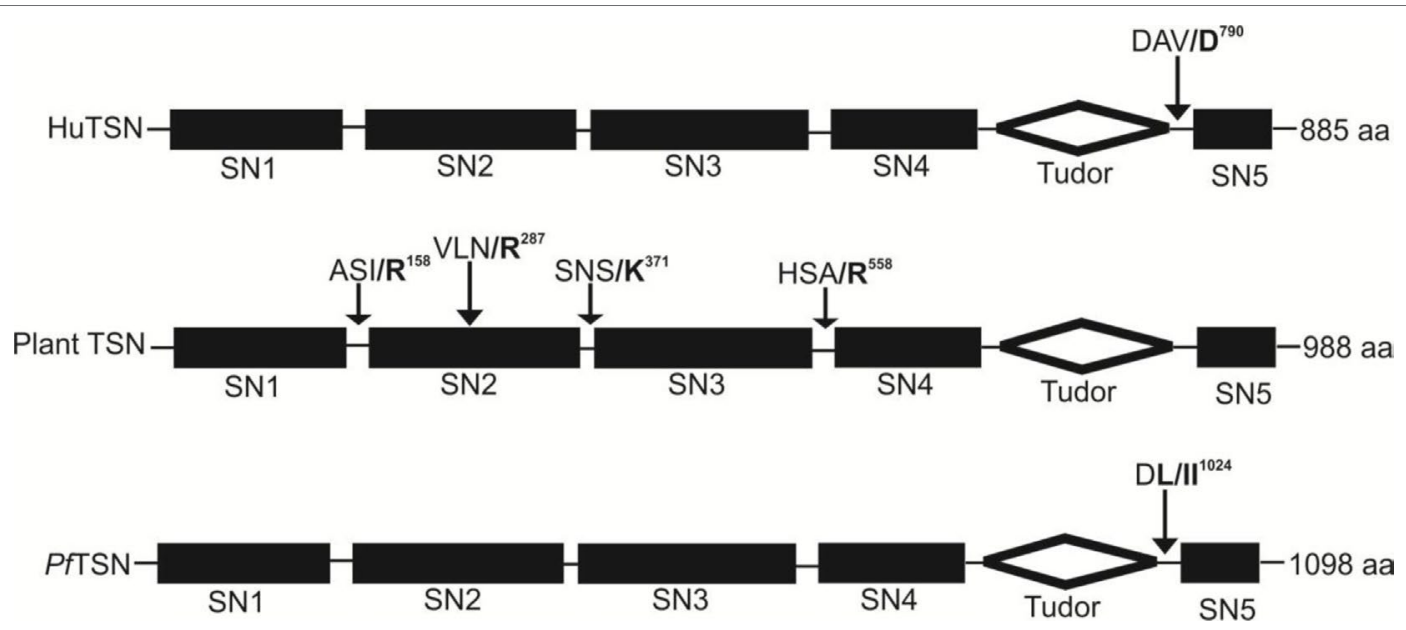

FIGURE 7 | Domains organization of Tudor staphylococcal nuclease (TSN) in human (AAA80488), Plant (CAL38976) and P. falciparum (PF3D7_1136300) and location of MCAs cleavage sites were indicated by arrows. The cleavage of TSN occurred before the bold residues in all presented TSN proteins.

area to investigate. In addition, the identification of protein targets other than PfTSN, which are cleaved by MCAs, and the molecular steps involved in activaltion of MCAs and TSN interaction will provide a fruitful avenue for future research.

\section{THERAPEUTIC POTENTIAL OF METACASPASES}

The unusual properties of metacaspases such as their distributions, catalytic site substrate specificity, biological functions, and their absence in humans make them a potential drug target. Several studies have supported this concept with the demonstration that protease inhibitors have potent in vitro an in vivo antipathogenic effects. In recent years, understanding of the protease repertoire of parasite has remarkably increased due to the advancement in biochemistry and structural biology. Therefore, considering the specificity of metacaspases activity in protozoan parasites and their absence in humans opens a new path to describe potential activators or inhibitors specific to metacaspases. The miltefosine, currently used for treating leishmaniasis, acts as an apoptotic stimulus and induces apoptosis by targeting metacaspases. Further, a report by Kumar et al. (2018) suggested that the chemical molecule that can target the biosynthetic pathway of metacaspases is probably used as an effective anti-leishmanial agent (Kumar et al., 2018). Similarly, Basmaciyan et al. (2018) reported three apoptosis pathways involving L. major MCA (LmjMCA) and its potent inhibitors: i) an apoptosis pathway in which LmjMCA is activated (induced by miltefosine); ii) a pathway in which LmjMCA is inhibited (amphotericin $\mathrm{B}$, curcumin, and $\left.\mathrm{H}_{2} \mathrm{O}_{2}\right)$; and iii) an LmjMCA-independent apoptosis pathway (pentamidine).

At present, it is not clearly known how protozoan metacaspases regulate the cell death in parasites, but it could be hypothesized that their conservation in protozoan presenting a long history of adaptation to environment reveals that metacaspases are key regulators of the parasite death or survival strategies. Therefore, in the near future, metacaspases could be considered as a potential drug target against parasitebased infectious diseases. Moreover, the development of potential inhibitors can either inactivate or activate the metacaspases pathway in order to favor parasite death either directly or through other mechanisms hindering one or several fundamental events caused by the metacaspases. In addition, inhibition of host cell apoptosis induced by a pathogen (Meslin et al., 2011) combined with induction of parasite apoptosis at the early stages of the infection is an integrative concept for future treatment that still needs to be elucidated deeply (Figure 4). Therefore, the approach for developing potent inhibitors against the parasite metacaspases, which can inhibit their activity further, can be useful for elucidating the therapeutic potential of protozoan metacaspases in detail.

\section{CONCLUSION}

Parasites get benefits from several virulence factors such as proteases, enzymes, co-factors, etc. For example, Plasmodium proteases characterized so far are involved in hemoglobin degradation (Falcipains and Plasmepsins) (Silva et al., 1996; Shenai et al., 2000; Pandey et al., 2005), invasion (Subtilase, Plasmepsin IX \& X) (Nasamu et al., 2017), egress and breakdown of RBC (SUB1 \& Plasmepsin II, X), trafficking pathway (Plasmepsin V) (Russo et al., 2010), and many more, which help parasites to survive in an intra- or extracellular host environment. Similarly, other parasite proteins such as Leishmania substilisin-like serine protease, which plays a role in promastigotes to amastigotes differentiation (Da Silva-Lopez et al., 2005), Presenilin 1 protease, involved in autophagy in L. major, which is involved in autophagy in $L$. major (Besteiro et al., 2007), Cruzain (cysteine protease) of T. cruzi, which helps in immune evasion (Doyle et al., 2011), 
etc., are found to be important for parasite physiology. Therefore, proteases are considered to be potent drug targets against parasites-based infectious diseases (Nuñez et al., 1998; Deponte, 2008). In this context, the function of nonmetazoan metacaspase-like proteases needs to be deeply explored as some recent findings suggest that these proteases are also important for the maintenance of physiology along with death and survival strategies of parasites (Jiménez-Ruiz et al., 2010; Deu, 2017). For instance, Trypanosoma and Leishmania metacaspases are increasingly being implicated as important players of PCD. Similarly, our recent report on PfMCA-2 reveals that the optimum level of PfMCA-2 was essential for parasite growth and cell viability. Further, our report also suggests that PfMCA-2 is important for stress regulation inside the cell (Vandana et al., 2018). Elucidating the mechanism behind the metacaspase-associated PCD is an important area of research, and it provides opportunities to target these proteases for potential drug discovery against parasite infectious diseases. It was also observed that specific metacaspase inhibitors disorient parasite morphology and reduce the cell viability and growth arrest, and also cause circumstantial cell death. Hence, such specific inhibitors will play an important role to understand the physiological significance of metacaspases-like proteases in disease pathogenesis and to identify them as promising candidates for drug development.

\section{REFERENCES}

Al-Olayan, E. M., Williams, G. T., and Hurd, H. (2002). Apoptosis in the malaria protozoan, Plasmodium berghei: a possible mechanism for limiting intensity of infection in the mosquito. Int. J. Parasitol. 32 (9), 1133-1143. doi: 10.1016/ S0020-7519(02)00087-5

Alvarez, V. E., Niemirowicz, G. T., and Cazzulo, J. J. (2012). The peptidases of Trypanosoma cruzi: digestive enzymes, virulence factors, and mediators of autophagy and programmed cell death. Biochim. Biophys. Acta. 1824 (1), 195206. doi: 10.1016/j.bbapap.2011.05.011

Aravind, L., and Koonin, E. V. (2002). Classification of the caspase-hemoglobinase fold: detection of new families and implications for the origin of the eukaryotic separins. Proteins 46, 355-367.

Arhel, N., and Kirchhoff, F. (2010). Host proteins involved in HIV infection: new therapeutic targets. Biochim. Biophys. Acta. 1802 (3), 313-321. doi: 10.1016/j. bbadis.2009.12.003

Arroyo, R., Cárdenas-Guerra, R. E., Figueroa-Angulo, E. E., Puente-Rivera, J., Zamudio-Prieto, O., and Ortega-López, J. (2015). Trichomonas vaginalis cysteine proteinases: iron response in gene expression and proteolytic activity. Bio. Med. Res. Int. 2015, 24. doi: 10.1155/2015/946787

Asplund-Samuelsson, J., Birgitta, B., and Larsson, J. (2012). Prokaryotic caspase homologs: phylogenetic patterns and functional characteristics reveal considerable diversity. PLoS One 7 (11), e49888. doi: 10.1371/journal. pone. 0049888

Atkinson, H. J., Babbitt, P. C., and Sajid, M. (2009). The global cysteine peptidase landscape in parasites. Trends Parasitol. 25 (12), 573-581. doi: 10.1016/j. pt.2009.09.006

Bagchi, S., Oniku, A. E., Topping, K., Mamhoud, Z. N., and Paget, T. A. (2012). Programmed cell death in Giardia. Parasitology 139 (7), 894-903. doi: 10.1017/ S003118201200011X

Basmaciyan, L., Azas, N., and Casanova, M. (2018). Different apoptosis pathways in Leishmania parasites. Cell Death Disc. 5 (1), 27. doi: 10.1038/s41420-018-0092-Z

Besteiro, S., Williams, R. A., Coombs, G. H., and Mottram, J. C. (2007). Protein turnover and differentiation in Leishmania. Int. J. Parasitol. 37 (10), 1063-1075. doi: 10.1016/j.ijpara.2007.03.008

\section{DATA AVAILABILITY}

All datasets generated for this study are included in the manuscript and the supplementary files.

\section{AUTHOR CONTRIBUTIONS}

$\mathrm{KV}$ and KP developed the idea, collected information, interpreted and reviewed the literature and wrote the article. $\mathrm{RD}, \mathrm{AK}$, and RT helped in the writing process. All the authors approved and reviewed the final version of the review article.

\section{FUNDING}

This work was supported by the Intramural grant of ICMR-NIMR and by the Ramalingaswami Fellowship, BT/HRD/35/02/2009, DBT sanctioned to KCP.

\section{ACKNOWLEDGMENTS}

$\mathrm{KV}$ would like to acknowledge Indian Council of Medical Research (ICMR) and Dr. B.R Ambedkar Centre for Biomedical Research, Delhi University for financial and academic support for the PhD program.

Bidle, K. D., and Bender, S. J. (2008). Iron starvation and culture age activate metacaspases and programmed cell death in the marine diatom Thalassiosira pseudonana. Eukaryotic Cell 7 (2), 223-236. doi: 10.1128/EC.00296-07

Carmona-Gutierrez, D., Fröhlich, K. U., Kroemer, G., and Madeo, F. (2010). Metacaspases are caspases. Doubt no more. Cell Death Differ 17, 377-378. doi: 10.1038/cdd.2009.198

Casanova, M., Gonzalez, I. J., Sprissler, C., Zalila, H., Dacher, M., Basmaciyan, L., et al. (2015). Implication of different domains of the Leishmania major metacaspase in cell death and autophagy. Cell Death Dis. 6 (10), e1933. doi: 10.1038/cddis.2015.288

Castanys-Muñoz, E., Brown, E., Coombs, G. H., and Mottram, J. C. (2012). Leishmania mexicana metacaspase is a negative regulator of amastigote proliferation in mammalian cells. Cell Death Dis. 3 (9), e385. doi: 10.1038/cddis.2012.113

Ch'Ng, J. H., Kotturi, S. R., Chong, A. G., Lear, M. J., and Tan, K. S. (2010). A programmed cell death pathway in the malaria parasite Plasmodium falciparum has general features of mammalian apoptosis but is mediated by clan CA cysteine proteases. Cell Death Dis. 1 (2), e26. doi: 10.1038/cddis.2010.2

Choi, C. J., and Berges, J. A. (2013). New types of metacaspases in phytoplankton reveal diverse origins of cell death proteases. Cell Death Dis. 4 (2), e490. doi: 10.1038/cddis.2013.21

Chose, O., Noël, C., Gerbod, D., Brenner, C., Viscogliosi, E., and Roseto, A. (2002). A form of cell death with some features resembling apoptosis in the amitochondrial unicellular organism Trichomonas vaginalis. Exp. Cell Res. 276 (1), 32-39. doi: 10.1006/excr.2002.5496

Chowdhury, S., Mukherjee, T., Chowdhury, S. R., Sengupta, S., Mukhopadhyay, S., Jaisankar, P., et al. (2014). Disuccinyl betulin triggers metacaspase-dependent endonuclease G-mediated cell death in unicellular protozoan parasite Leishmania donovani. Antimicrob. Agents Chemother. 58 (4), 2186-2201. doi: 10.1128/AAC.02193-13

Da Silva-Lopez, R. E., Coelho, M. P., and De Simone, S. G. (2005). Characterization of an extracellular serine protease of Leishmania (Leishmania) amazonensis. Parasitology 131 (1), 85-96. doi: 10.1017/S0031182004006675

de Castro, E., Reus, T. L., de Aguiar, A. M., and Ávila, A. R. (2017). Procaspaseactivating compound-1 induces apoptosis in Trypanosoma cruzi. Apoptosis 22 (12), 1564-1577. doi: 10.1007/s10495-017-1428-5 
Deponte, M. (2008). Programmed cell death in protists. Biochim. Biophys. Acta, Mol. Cell. Res. 1783 (7), 1396-1405. doi: 10.1016/j.bbamcr.2008.01.018

Deu, E. (2017). Proteases as antimalarial targets: strategies for genetic, chemical, and therapeutic validation. FEBS J. 284 (16), 2604-2628. doi: 10.1111/ febs. 14130

Dit Frey, N. F., Muller, P., Jammes, F., Kizis, D., Leung, J., Perrot-Rechenmann, C., et al. (2010). The RNA binding protein Tudor-SN is essential for stress tolerance and stabilizes levels of stress-responsive mRNAs encoding secreted proteins in Arabidopsis. Plant Cell 22 (5), 1575-1591. doi: 10.1105/tpc.109.070680

Dolai, S., Pal, S., Yadav, R. K., and Adak, S. (2011). Endoplasmic reticulum stressinduced apoptosis in Leishmania through Ca2+-dependent and caspaseindependent mechanism. J. Biol. Chem. 286 (15), 13638-13646. doi: 10.1074/ jbc.M110.201889

Domínguez-Fernández, T., Rodriguez, M. A., Monroy, V. S., García, C. G., Medel, O., and Ishiwara, D. G. P. (2018). A calpain-like protein is involved in the execution phase of programmed cell death of Entamoeba histolytica. Front. Cell Infection Microbiol. 8, 339. doi: 10.3389/fcimb.2018.00339

Doyle, P. S., Zhou, Y. M., Hsieh, I., Greenbaum, D. C., McKerrow, J. H., and Engel, J. C. (2011). The Trypanosoma cruzi protease cruzain mediates immune evasion. PLoS Pathog. 7 (9), e1002139. doi: 10.1371/journal.ppat.1002139

Dubios, K. N., Abodeely, M., Sakanari, J., Craik, C. S., Lee, M., McKerrow, J. H., et al. (2008). Identification of the major cysteine protease of Giardia and its role in encystation. J. Biol. Chem. 283 (26),18024-18031. doi: 10.1074/jbc. M802133200

Elmore, S. (2007). Apoptosis: a review of programmed cell death. Toxicol. Pathol. 35 (4), 495-516. doi: 10.1080/01926230701320337

Engelbrecht, D., and Coetzer, T. L. (2015). Sunlight inhibits growth and induces markers of programmed cell death in Plasmodium falciparum in vitro. Malar J. 14 (1), 378. doi: 10.1186/s12936-015-0867-0

Engstler, M., Thilo, L., Weise, F., Grünfelder, C. G., Schwarz, H., Boshart, M., et al. (2004). Kinetics of endocytosis and recycling of the GPI-anchored variant surface glycoprotein in Trypanosoma brucei. J. Cell Sci. 117 (7), 1105-1115. doi: $10.1242 /$ jcs.00938

González, I. J., Desponds, C., Schaff, C., Mottram, J. C., and Fasel, N. (2007). Leishmania major metacaspase can replace yeast metacaspase in programmed cell death and has arginine-specific cysteine peptidase activity. Int. J. Parasitol. 37 (2), 161-172. doi: 10.1016/j.ijpara.2006.10.004

Gutierrez-Beltran, E., Denisenko, T. V., Zhivotovsky, B., and Bozhkov, P. V. (2016). Tudor staphylococcal nuclease: biochemistry and functions. Cell Death Differ 23 (11), 1739-1748. doi: 10.1038/cdd.2016.93

Hachmann, J., Snipas, S. J., Van Raam, B. J., Cancino, E. M., Houlihan, E. J., Poreba, M., et al. (2012). Mechanism and specificity of the human paracaspase MALT1. Biochem. J. 443 (1), 287-295. doi: 10.1042/BJ20120035

Helms, M. J., Ambit, A., Appleton, P., Tetley, L., Coombs, G. H., and Mottram, J. C. (2006). Bloodstream form Trypanosoma brucei depend upon multiple metacaspases associated with RAB11-positive endosomes. J. Cell Sci. 119 (6), 1105-1117. doi: $10.1242 /$ jcs. 02809

Hulpiau, P., Driege, Y., Staal, J., and Beyaert, R. (2016). MALT1 is not alone after all: identification of novel paracaspases. Cell Mol. Life Sci. 73 (5), 1103-1116. doi: 10.1007/s00018-015-2041-9

Jaworski, M., and Thome, M. (2016). The paracaspase MALT1: biological function and potential for therapeutic inhibition. Cell Mol. Life Sci. 73 (3), 459-473. doi: 10.1007/s00018-015-2059-z

Jiménez-Ruiz, A., Alzate, J. F., MacLeod, E. T., Lüder, C. G. K., Fasel, N., and Hurd, H. (2010). Apoptotic markers in protozoan parasites. Parasites Vectors, 3 (1), 104. doi: 10.1186/1756-3305-3-104

Kanhaiya, K., Czeizler, E., Gratie, C., and Petre, I. (2017). Controlling directed protein interaction networks in cancer. Sci. Rep. 7 (1), 10327. doi: 10.1038/ s41598-017-10491-y

Khademvatan, S., Gharavi, M. J., and Saki, J. (2011). Miltefosine induces metacaspase and PARP genes expression in Leishmania infantum. Braz. J. Infect. Dis. 15 (5), 442-448. doi: 10.1590/S1413-86702011000500005

Klemenčič, M., and Funk, C. (2018). Structural and functional diversity of caspase homologues in non-metazoan organisms. Protoplasma 255 (1), 387-397. doi: 10.1007/s00709-017-1145-5

Klemenčič, M., and Funk, C. (2019). Evolution and structural diversity of metacaspases. J. Exp. Botany 70 (7), 2039-2047. doi: 10.1093/jxb/erz082
Kosec, G., Alvarez, V., and Cazzulo, J. J. (2006). Cysteine proteinases of Trypanosoma cruzi: from digestive enzymes to programmed cell death. Biocell 30 (3), 479-490.

Kumar, A., Pandey, S. C., and Samant, M. (2018). Slow pace of antileishmanial drug development. Parasitol. Open 4 (e4), 1-11. doi: 10.1017/pao.2018.1

Laverrière, M., Cazzulo, J. J., and Alvarez, V. E. (2012). Antagonic activities of Trypanosoma cruzi metacaspases affect the balance between cell proliferation, death and differentiation. Cell Death Differ. 19 (8), 1358. doi: 10.1038/ cdd.2012.12

Lee, N., Gannavaram, S., Selvapandiyan, A., and Debrabant, A. (2007). Characterization of metacaspases with trypsin-like activity and their putative role in programmed cell death in the protozoan parasite Leishmania. Eukaryot. Cell 6 (10), 1745-1757. doi: 10.1128/EC.00123-07

Li, M., Wang, H., Liu, J., Hao, P., Ma, L., and Liu, Q. (2016). The apoptotic role of metacaspase in Toxoplasma gondii. Front. Microbiol. 6, 1560. doi: 10.3389/ fmicb. 2015.01560

Machado, M. F., Marcondes, M. F., Juliano, M. A., McLuskey, K., Mottram, J. C., Moss, C. X., et al. (2013). Substrate specificity and the effect of calcium on Trypanosoma brucei metacaspase 2. FEBS J. 280 (11), 2608-2621. doi: 10.1111/ febs. 12248

McIlwain, D. R., Berger, T., and Mak, T. W. (2013). Caspase functions in cell death and disease. Cold Spring Harb. Perspect. Biol.5 (4), a008656.

McLuskey, K., and Mottram, J. C. (2015). Comparative structural analysis of the caspase family with other clan CD cysteine peptidases. Biochem. J. 466 (2), 219-232. doi: 10.1042/BJ20141324

McLuskey, K., Moss, C. X., and Mottram, J. C., (2014). "Purification, characterization, and crystallization of Trypanosoma metacaspases," in Caspases, Paracaspases, and Metacaspases. Methods in Molecular Biology (Methods and Protocols), vol. 1133. Eds. P. V Bozhkov and G. Salvesen (New York, NY: Humana Press), 203-221. doi: 10.1007/978-1-4939-0357-3_13

McLuskey, K., Rudolf, J., Proto, W. R., Isaacs, N. W., Coombs, G. H., Moss, C. X. et al. (2012). Crystal structure of a Trypanosoma brucei metacaspase. Proc. Natl. Acad. Sci. U. S. A. 109 (19), 7469-7474. doi: 10.1073/pnas.1200885109

Meslin, B., Barnadas, C., Boni, V., Latour, C., De Monbrison, F., Kaiser, K., et al. (2007). Features of apoptosis in Plasmodium falciparum erythrocytic stage through a putative role of PfMCA1 metacaspase-like protein. J. Infect. Dis. 195 (12), 1852-1859. doi: $10.1086 / 518253$

Meslin, B., Zalila, H., Fasel, N., Picot, S., and Bienvenu, A. L. (2011). Are protozoan metacaspases potential parasite killers? Parasites Vectors 4 (1), 26. doi: 10.1186/1756-3305-4-26

Minina, E. A., Coll, N. S., Tuominen, H., and Bozhkov, P. V. (2017). Metacaspases versus caspases in development and cell fate regulation. Cell Death Differ. 24 (8), 1314. doi: 10.1038/cdd.2017.18

Moss, C. X., Westrop, G. D., Juliano, L., Coombs, G. H., and Mottram, J. C. (2007). Metacaspase 2 of Trypanosoma brucei is a calcium-dependent cysteine peptidase active without processing. FEBS Lett. 581 (29), 5635-5639. doi: 10.1016/j.febslet.2007.11.009

Mottram, J. C., Helms, M. J., Coombs, G. H., and Sajid, M. (2003). Clan CD cysteine peptidases of parasitic protozoa. Trends Parasitol. 19 (4), 182-187. doi: 10.1016/S1471-4922(03)00038-2

Mutai, B. K., and Waitumbi, J. N. (2010). Apoptosis stalks Plasmodium falciparum maintained in continuous culture condition. Malar. J. 9 (3), S6. doi: 10.1186/1475-2875-9-S3-S6

Nasamu, A. S., Glushakova, S., Russo, I., Vaupel, B., Oksman, A., Kim, A. S., et al. (2017). Plasmepsins IX and X are essential and druggable mediators of malaria parasite egress and invasion. Science 358 (6362), 518-522. doi: 10.1126/science. aan1478

Nasirudeen, A. M. A., and Tan, K. S. (2004). Caspase-3-like protease influences but is not essential for DNA fragmentation in Blastocystis undergoing apoptosis. Eur. J. Cell Biol. 83 (9), 477. doi: 10.1078/0171-9335-00411

Nuñez, G., Benedict, M. A., Hu, Y., and Inohara, N. (1998). Caspases: the proteases of the apoptotic pathway. Oncogene 17 (25), 3237. doi: 10.1038/ sj.onc. 1202581

Pandey, K. C., Wang, S. X., Sijwali, P. S., Lau, A. L., McKerrow, J. H., and Rosenthal, P. J. (2005). The Plasmodium falciparum cysteine protease falcipain-2 captures its substrate, hemoglobin, via a unique motif. Proc. Natl. Acad. Sci. U. S. A. 102 (26), 9138-9143. doi: 10.1073/pnas.0502368102 
Peña, M. S., Cabral, G. C., Fotoran, W. L., Perez, K. R., and Stolf, B. S. (2017). Metacaspase-binding peptide inhibits heat shock-induced death in Leishmania (L). amazonensis. Cell Death Dis. 8 (3), e2645. doi: 10.1038/cddis.2017.59

Picot, S., Burnod, J., Bracchi, V., Chumpitazi, B. F. F., and Ambroise-Thomas, P. (1997). Apoptosis related to chloroquine sensitivity of the human malaria parasite Plasmodium falciparum. Trans. R. Soc. Trop. Med. Hyg. 91 (5), $590-$ 591. doi: 10.1016/S0035-9203(97)90039-0

Pop, C., and Salvesen, G. S. (2009). Human caspases: activation, specificity, and regulation. J. Biol. Chem. 284 (33), 21777-21781. doi: 10.1074/jbc.R800084200

Proto, W. R., Castanys-Munoz, E., Black, A., Tetley, L., Moss, C. X., Juliano, L., et al. (2011). Trypanosoma brucei Metacaspase 4 is a Pseudopeptidase and a Virulence factor. J. Biol. Chem. 286 (46), 39914-39925. doi: 10.1074/jbc.M111.292334

Rahman, M. M. (2010). Biochemical analysis of type II Metacaspase (mcII-Pa). Masters Thesis. Swedish University of Agricultural Sciences.

Rathore, S., Datta, G., Kaur, I., Malhotra, P., and Mohmmed, A. (2015). Disruption of cellular homeostasis induces organelle stress and triggers apoptosis like celldeath pathways in malaria parasite. Cell Death Dis. 6 (7), e1803. doi: 10.1038/ cddis. 2015.142

Rawlings, N. D., Barrett, A. J., Thomas, P. D., Huang, X., Bateman, A., and Finn, R. D. (2017). The MEROPS database of proteolytic enzymes, their substrates and inhibitors in 2017 and a comparison with peptidases in the PANTHER database. Nucleic Acids Res. 46 (D1), D624-D632.

Rochefort, G. Y. (2014). The osteocyte as a therapeutic target in the treatment of osteoporosis. Ther. Adv. Musculoskelet. Dis. 6 (3), 79-91. doi: 10.1177/1759720X14523500

Russo, I., Babbitt, S., Muralidharan, V., Butler, T., Oksman, A., and Goldberg, D. E. (2010). Plasmepsin V licenses Plasmodium proteins for export into the host erythrocyte. Nature 463 (7281), 632. doi: 10.1038/nature08726

Shadab, M., Jha, B., Asad, M., Deepthi, M., Kamran, M., and Ali, N. (2017). Apoptosislike cell death in Leishmania donovani treated with KalsomeTM10, a new liposomal amphotericin B. PloS One 12 (2), e0171306. doi: 10.1371/journal.pone.0171306

Shenai, B. R., Sijwali, P. S., Singh A., and Rosenthal, P. J. (2000). Characterization of native and recombinant falcipain-2, a principal trophozoite cysteine protease and essential hemoglobinase of Plasmodium falciparum. J. Biol. Chem. 275 (37), 29000-29010. doi: 10.1074/jbc.M004459200

Shrestha, A., and Megeney, L. A. (2012). The non-death role of metacaspase proteases. Front. Oncol. 2, 78. doi: 10.3389/fonc.2012.00078

Silva, A. M., Lee, A. Y., Gulnik, S. V., Maier, P., Collins, J., Bhat, T. N., et al. (1996). Structure and inhibition of plasmepsin II, a hemoglobin-degrading enzyme from Plasmodium falciparum. Proc. Natl. Acad. Sci. U. S. A. 93 (19), $10034-$ 10039. doi: 10.1073/pnas.93.19.10034

Sundström, J. F., Vaculova, A., Smertenko, A. P., Savenkov, E. I., Golovko, A., Minina, E., et al. (2009). Tudor staphylococcal nuclease is an evolutionarily conserved component of the programmed cell death degradome. Nat. Cell Biol. 11 (11), 1347. doi: 10.1038/ncb1979

Szallies, A., Kubata, B. K., and Duszenko, M. (2002). A metacaspase of Trypanosoma brucei causes loss of respiration competence and clonal death in the yeast Saccharomyces cerevisiae. FEBS Lett. 517 (1-3), 144-150. doi: 10.1016/ S0014-5793(02)02608-X

Tsiatsiani, L., Van Breusegem, F., Gallois, P., Zavialov, A., Lam, E., and Bozhkov, P. V. (2011). Metacaspases. Cell Death Differ 18, 1279-1288. doi: 10.1038/ cdd. 2011.66

Uren, A. G., O’Rourke, K., Aravind, L., Pisabarro, M. T., Seshagiri, S., Koonin, E. V., et al. (2000). Identification of paracaspases and metacaspases: two ancient families of caspase-like proteins, one of which plays a key role in MALT lymphoma. Mol. Cell 6 (4), 961-967. doi: 10.1016/S1097-2765(00)00094-0

van Creveld, S. G., Ben-Dor, S., Mizrachi, A., Alcolombri, U., Hopes, A., Mock, T., et al. (2018). A redox-regulated type III metacaspase controls cell death in a marine diatom. bioRxiv 444109. doi: 10.1101/444109

Vandana, Singh, A.P., Singh, J., Sharma, R., Akhter, M., Mishra, P. K., Saxena, A. K., et al. (2018). Biochemical characterization of unusual cysteine protease of $P$. falciparum, metacaspase-2 (MCA-2). Mol. Biochem. Parasitol. 220, 28-41. doi: 10.1016/j.molbiopara.2018.01.001

Vercammen, D., Van De Cotte, B., De Jaeger, G., Eeckhout, D., Casteels, P., Vandepoele, K., et al. (2004). Type II metacaspases Atmc4 and Atmc9 of Arabidopsis thaliana cleave substrates after arginine and lysine. J. Biol. Chem. 279 (44), 45329-45336. doi: 10.1074/jbc.M406329200

Walker, N. P. C., Talanian, R. V., Brady, K. D., Dang, L. C., Bump, N. J., Ferenz, C. R., et al. (1994). Crystal-structure of the cysteine protease interleukin-1-betaconverting enzyme-a (P20/P10)(2) homodimer. Cell 78, 343-352.

Zalila, H., González, I. J., El-Fadili, A. K., Delgado, M. B., Desponds, C., Schaff, C., et al. (2011). Processing of metacaspase into a cytoplasmic catalytic domain mediating cell death in Leishmania major. Mol. Microbiol. 79 (1), 222-239. doi: 10.1111/j.1365-2958.2010.07443.x

Conflict of Interest Statement: The authors declare that the research was conducted in the absence of any commercial or financial relationships that could be construed as a potential conflict of interest.

Copyright (c) 2019 Vandana, Dixit, Tiwari, Katyal and Pandey. This is an openaccess article distributed under the terms of the Creative Commons Attribution License (CC BY). The use, distribution or reproduction in other forums is permitted, provided the original author(s) and the copyright owner(s) are credited and that the original publication in this journal is cited, in accordance with accepted academic practice. No use, distribution or reproduction is permitted which does not comply with these terms. 